\title{
Influence of Masticatory Functional Loss on the Remodeling of Alveolar Bone in Rats
}

\author{
Kei Ogawa ${ }^{1,2}$, Takamichi Yamada ${ }^{3}$, Norimitsu Hirai ${ }^{1}$, Wataru Morita ${ }^{1}$, Yurie Okada ${ }^{1}$, \\ Seiko Yamamoto-Nemoto ${ }^{1,2}$, Takehiko Shimizu ${ }^{1,2}$ \\ ${ }^{1}$ Department of Pediatric Dentistry, Nihon University School of Dentistry at Matsudo, Matsudo, Chiba, Japan \\ ${ }^{2}$ Nihon University Research Institute of Oral Science, Matsudo, Chiba, Japan \\ ${ }^{3}$ Department of Pediatric Dentistry, Nihon University Graduate School of Dentistry at Matsudo, Matsudo, Chiba, Japan \\ Email: ogawa.kei@nihon-u.ac.jp
}

How to cite this paper: Ogawa, K., Yamada, T., Hirai, N., Morita, W., Okada, Y., Yamamoto-Nemoto, S. and Shimizu, T. (2020) Influence of Masticatory Functional Loss on the Remodeling of Alveolar Bone in Rats. Open Journal of Stomatology, 10, 341-359.

https://doi.org/10.4236/ojst.2020.1012032

Received: November 3, 2020

Accepted: November 27, 2020

Published: November 30, 2020

Copyright (c) 2020 by author(s) and Scientific Research Publishing Inc. This work is licensed under the Creative Commons Attribution International License (CC BY 4.0).

http://creativecommons.org/licenses/by/4.0/

\begin{abstract}
There is plenty of literature on masticatory function and its impact on maxillofacial development. However, the influence of masticatory hypofunction on bone turnover in the alveolar bone has hardly been studied. This study aimed to clarify the influence of tooth loss and soft diet on the alveolar bone turnover during the growth period. Three-week-old Wistar rats were randomly divided into the following three groups: Hard diet group (rats raised on solid standard diet), Powder diet group (rats raised on powdered standard feed diet), and Extraction group (rats raised on powdered standard diet with maxillary molars extraction). BV, BMC, and BMD in the cancellous bone of M1 were measured using micro-CT analysis. To analyze the histological bone turnover, we prepared non-decalcified thin sections of alveolar cancellous bone when rats were 20 weeks old. On three-dimensional constructed images, the experimental groups (the Powder diet and Extraction groups) showed expansion of the medullary cavity of the interradicular septum of the first molar compared to controls (the Hard diet group). BV, BMC, and BMD were significantly lower in the experimental groups, with the difference from controls being greater in the Extraction group. On histomorphometric analysis, the bone mass parameters, bone formation parameters, and bone mineralization parameters were significantly lower in the experimental groups compared to controls. The bone resorption parameters were significantly higher in the experimental groups. From this study, we found that soft diet and tooth loss might worsen the bone microstructure, reduce osteogenesis, and promote bone resorption in alveolar bone.
\end{abstract}

\section{Keywords}

Rats, Alveolar Bone, Powder Diet, Extraction of Molars, Bone 
Histomorphometry

\section{Introduction}

Amid the growing popularity of processed food and soft food diets, children increasingly prefer softer foods that require fewer mastication iterations and less time for mastication. This preference for softer foods may affect the child's maxillofacial development, narrowing the jaw and tooth rows and resulting in malocclusion. Another pediatric dental problem is masticatory hypofunction, which may be related to congenital hypodentia or premature tooth loss caused by dental trauma or dental caries. When chewing food, children with masticatory hypofunction apply less mechanical force to their jaw and facial muscles than otherwise, which significantly affects physical growth. Thus, the consistency of food ingested and the condition of the oral cavity are critical factors in ensuring appropriate masticatory stimulation to the maxillofacial and masticatory muscles.

There is plenty of literature on masticatory function and its impact on maxillofacial development. Moss et al. [1] argued that biological functions are related to their corresponding skeletal components. In humans, they claimed that components of the mandible powerfully shape the performance of masticatory muscles. Specifically, the temporalis shapes the coronoid process, the masseter muscle shapes the mandibular condyle, and the medial and lateral pterygoid muscles shape the gonial angle. There are many experiments on reduced chewing stimulation with a soft diet. These experiments showed a reduction in maxillary width, mandibular height and mandibular condyle thickness, and expansion of the size of the mandibular angle [2] [3] [4] [5] [6]. Similarly, our previous research showed masticatory hypofunction in rats, due to extraction of maxillary molars and a diet of powdered feed, was associated with reduced mandibular volume, bone density, mandibular height, and mandibular condyle thickness [7] [8]. Additionally, we indicated that reduced masticatory function led to deformation and cellular disorders in the condylar cartilage, and diminished ossification and heightened bone resorption in the secondary cancellous bone of the condylar head, resulting in slower ossification, less bone volume, and reduced trabecular thickness [9]. There are also reports on the effects of occlusal stimuli on the alveolar bone: In rats, a soft diet, tooth extraction or the insertion of a bite-opening appliance were associated with a thinner [10] [11] [12] [13] and higher alveolar process [6] [10] [13] [14] [15], and sparser mandibular alveolar architecture [15] [16]. Furthermore, it is reported that new bone was added at the outer layer of the alveolar bone, with an atrophic change in the periodontal ligament fibers [17].

Bone remodeling is a process in which osteoclasts break down bone tissue and osteoblasts build new bone tissue. Dental practice will benefit from a better understanding of how reduced masticatory stimuli and masticatory hypofunction 
affect bone turnover in the alveolar bone. To our knowledge, bone mass and the bone density of alveolar bone have been reported; however, the bone turnover of alveolar bone has been hardly studied.

Accordingly, the purpose of this present study was to clarify the influence of tooth loss and soft diet on the alveolar bone turnover during the growth period. To assess bone remodeling, we used the alveolar bone volume, mineral content, alveolar density, which we measured using micro-CT analysis. To analyze bone turnover at a histological level, we prepared non-decalcified thin sections of alveolar cancellous bone for histomorphometric analysis.

\section{Materials and Methods}

\subsection{Experimental Animals}

We purchased three-week-old Wistar rats (21 male rats) from Sankyo Lab Service Co., Ltd., and randomly divided them into the following three groups (seven rats each): 1) Hard diet group: These rats were raised on solid standard feed (MF, Oriental Yeast Co., Ltd, Tokyo, Japan). 2) Powder diet group: These rats were raised on powdered standard feed. 3) Extraction group: When the rats were 4 weeks old, we intraperitoneally administered thiamylal sodium $(15 \mathrm{mg} / \mathrm{kg}$; Kyorin Pharmaceutical Co., Ltd., Tokyo, Japan) and used a spoon excavator under general anesthesia to dislocate and extract all the maxillary molars on both sides. After extraction, we raised the rats on powdered standard feed.

The animals were raised in the rat facility at the Laboratory Animal Research Center of our university with a room temperature of $24^{\circ} \mathrm{C} \pm 1^{\circ} \mathrm{C}$; a constant humidity level of $65 \% \pm 5 \%$; and a 12 -hour light/dark cycle. All rats were given distilled water to drink and had free access to feed and water.

Tetracycline (20 mg/kg; Sigma-Aldrich, St. Louis, MO, USA) and calcein (10 $\mathrm{mg} / \mathrm{kg}$; Sigma-Aldrich) were subcutaneously injected seven days and one day before euthanasia, respectively, in order to double-label the bones. At the age of 20 weeks, the rats were euthanized with carbon dioxide gas, and the mandibles on both sides were removed.

The experimental schedule and procedures are summarized in Figure 1.

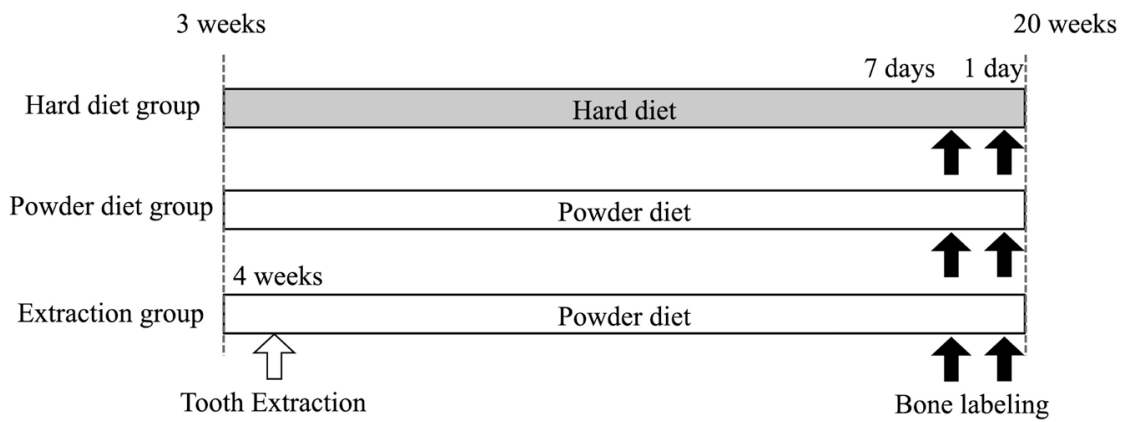

Figure 1. Time-schedule of the experiment. When the rats were 4 weeks old, we extracted all the maxillary molars on both sides (white arrow). Tetracycline and calcein were subcutaneously injected seven days and one day before euthanasia, respectively, in order to double-label the bones (black arrows). 
This study was approved by the Ethics Committee of the Nihon University School of Dentistry at Matsudo (animal experiment approval number: AP13MD020). The study design adhered to ARRIVE guidelines and was approved by the appropriate ethics review board.

\subsection{Measurement of Body Weight}

The rats' body weights were measured weekly from the age of 3 weeks until the age of 20 weeks.

\subsection{Micro-CT Analysis}

We imaged the extracted right mandibular body using micro-CT (R_mCT2, Rigaku, Tokyo, Japan). The imaging conditions were as follows: tube voltage, 90 $\mathrm{kV}$; tube current, $160 \mu \mathrm{A}$; magnification, $2 \times$; measurement time, 3 minutes; Field of View, $3 \mathrm{~mm}$. Slice images were constructed in three dimensions using 3-D construction analysis software (TRI/3D-BON, Ratoc System Engineering Co, Tokyo, Japan). The bone volume (BV), bone mineral content (BMC), and bone mineral density (BMD) were measured with a range of $600 \mu \mathrm{m} \times 600 \mu \mathrm{m} \times 600$ $\mu \mathrm{m}$ in the cancellous bone of interradicular septum of the first molar (Figure 2).

\subsection{Histological Preparation}

We extracted the left mandibular body of each rat and fixed it in $70 \%$ ethanol. We discarded the soft tissue and then immersed the bone for 4 days in Villanueva bone stain without decalcification. After dehydrating with ethanol, we wrapped the mandibular body with a methyl methacrylate resin. We cut the sagittal sections of the alveolar part into sections of $5 \mu \mathrm{m}$ thickness using a microtome and prepared non-decalcified thin-slice specimens for histological observation.
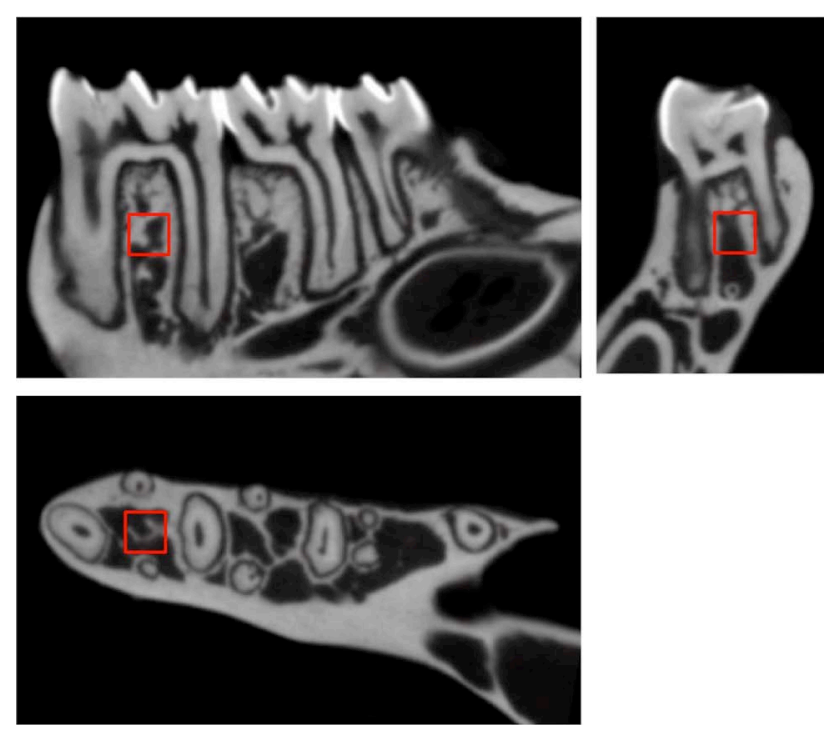

Figure 2. BV, BMC, and BMD were measured at the range of $600 \mu \mathrm{m} \times 600 \mu \mathrm{m} \times 600 \mu \mathrm{m}$ in the cancellous bone of the interradicular septum in the first molar. The square indicates the measurement range. 


\subsection{Bone Histomorphometry of Cancellous Bone}

The cancellous bone was measured at the interradicular septum of the lower first molar: a region of 1217 to $1643 \mathrm{~mm}^{2}$ was selected. The following parameters were measured: Interradicular septum volume (I.S.V), interradicular septal width (I.S.Wi), osteoid thickness (O.Th), osteoid surface/bone surface (OS/BS), eroded surface/bone surface (ES/BS), quiescent surface/bone surface (QS/BS), osteoblast number/bone surface (N.Ob/BS), osteoclast number/bone surface (N.Oc/BS), mineral apposition rate (MAR), mineralizing surface/bone surface (MS/BS) mineralization lag time (Mlt), bone formation rate/bone surface (BFR/BS) and bone formation rate/interradicular septum volume (BFR/I.S.V). For the N.Oc, mononuclear osteoclasts (N.Mo.Oc) and multinuclear osteoclasts (N.Mu.Oc) were measured separately.

\subsection{Statistical Analysis}

All statistical analyses were performed using SPSS (IBM Corp., Armonk, NY). All data are expressed as the mean \pm SD. ANOVA was used to determine differences between multiple groups, while differences between individual groups were determined by Tukey's test. A $p$ value equal to or less than 0.05 was considered to be statistically significant.

\section{Results}

\subsection{Body Weight}

The mean body weight increased over time for all groups, and no significant differences were found among the groups at any point in time (Figure 3).

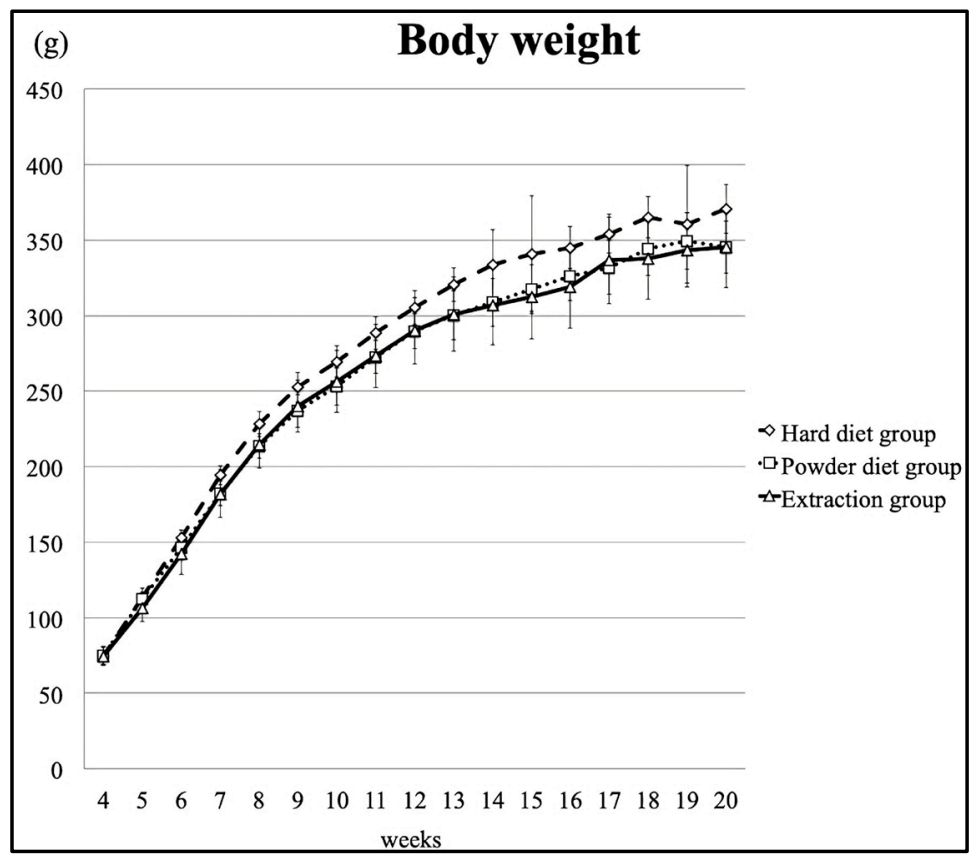

Figure 3. Body weight of the rats, by weeks. The body weight increased with age, and no ignificant difference was found between the groups at any point in time. 


\subsection{CT-Based Assessment of the Alveolar Bone}

In the three-dimensional constructed images, both the Extraction group and the Powder diet group showed expansion of the bone medullary cavity of the interradicular septum of the first molar compared to the Hard diet group. The tendency was recognized conspicuously in the Extraction group (Figure 4).

$\mathrm{BV}$ in the cancellous bone of interradicular septum of the first molar was 0.18 $\pm 0.02 \mathrm{~mm}^{3}$ for the Hard diet group, $0.13 \pm 0.01 \mathrm{~mm}^{3}$ for the Powder diet group, and $0.07 \pm 0.01 \mathrm{~mm}^{3}$ for the Extraction group. BMC in the cancellous bone of interradicular septum of the first molar was $0.126 \pm 0.031 \mathrm{mg}$ for the Hard diet group, $0.063 \pm 0.011 \mathrm{mg}$ for the Powder diet group, and $0.027 \pm 0.008 \mathrm{mg}$ for the Extraction group. BMD in the cancellous bone of interradicular septum of the first molar was $674.21 \pm 108.79 \mathrm{mg} / \mathrm{cm}^{3}$ for the Hard diet group, $471.25 \pm 51.21$ $\mathrm{mg} / \mathrm{cm}^{3}$ for the Powder diet group, and $369.07 \pm 64.02 \mathrm{mg} / \mathrm{cm}^{3}$ for the Extraction group. The Extraction group and the Powder diet group showed significantly lesser values than the Hard diet group for all three parameters. The BV and BMC in the Extraction group were significantly lesser than those in the Powder diet group (Figure 5).

\subsection{Histological Observation}

Regarding the inner architecture of the interradicular septa, the specimens from

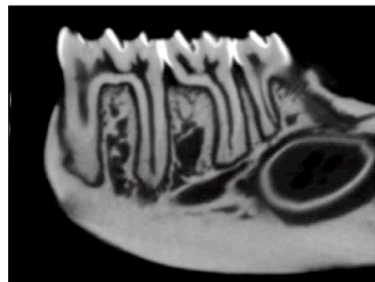

Hard diet group

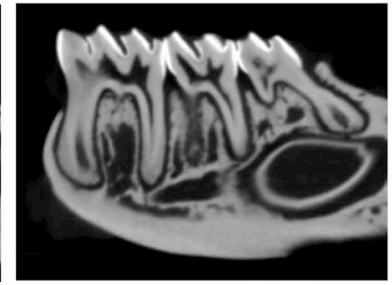

Powder diet group

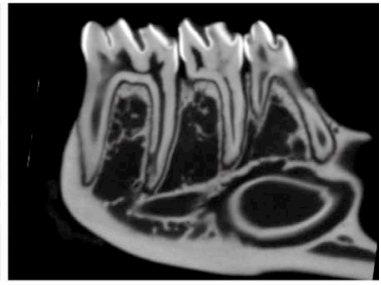

Extraction group

Figure 4. Micro-CT images. Both the powder diet group and the extraction group showed expansion of the bone medullary cavity of the interradicular septum compared to the Hard diet group. The tendency was recognized conspicuously in the Extraction group.

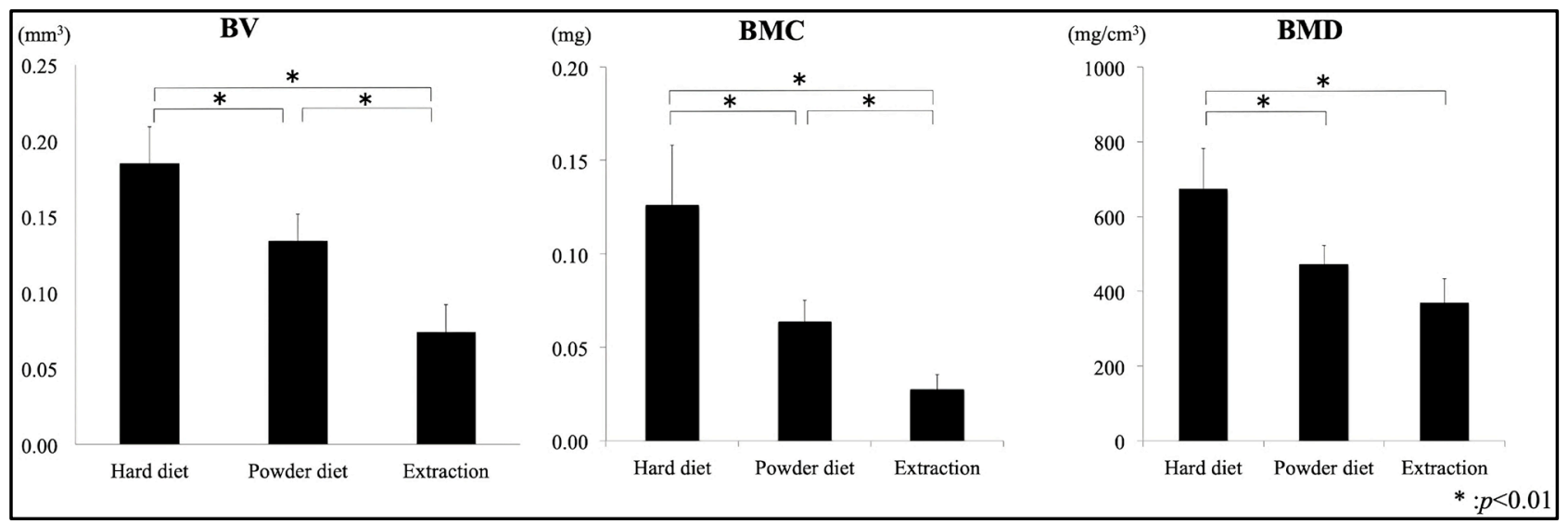

Figure 5. BV, BMC, and BMD in the micro-CT analysis. Data are presented as mean \pm standard deviation $\left({ }^{*} p<0.01\right)$. BV: bone volume. BMC: bone mineral content. BMD: bone mineral density. 
the Hard diet group had narrow medullary cavities with extensive trabecular distribution, those from the Powder diet group had wide medullary cavities with isolated trabecular clusters, and those from the Extraction group had markedly wide medullary cavities with several thin trabecular beams. Among the specimens from the Hard diet group, the upper section of the septal outline (immediately below the crown) was thick and continuous (without any breaks), and the overall outline was proportionate to that of the root branches on the other side of Sharpey fibers. In contrast, in the specimens from the two experimental groups, the upper section of the outline was thick, but the sections further down the branches of the root were thin, with breaks in some places. This result was particularly conspicuous in the specimens from the Extraction group (Figure 6).

Hard diet group: The septal outline is proportionate to that of the root branches on the other side of the Sharpey's fibers. The internal architecture features narrow medullary cavities with extensive trabecular distribution.

Powder diet group: The center of the septal apex is bifurcated. The upper section of the outline (directly underneath the crown) is thick, but the lines are thinner in the lower sections along the root. The internal architecture features wide medullary cavities with isolated trabecular clusters.

Extraction group: The center of the septal apex is trifurcated. The upper section of the outline (directly underneath the crown) is thick, but the lines running down the root are conspicuously thin and broken in several places. The internal architecture features markedly wide medullary cavities with several thin trabecular beams.

The specimens from the experimental groups had more polynuclear osteoclasts than those from the control group, suggesting the presence of deeper resorption cavities (Figure 7(a)). The osteoblasts in the extraction specimens were different, based on the classification system used by Villanueva et al. [18] from those in the Hard diet and Powder diet specimens. The osteoblasts in the Hard diet specimens were identified as type II (cuboidal) osteoblasts based on the presence of cubic cytoplasm. The cytoplasm was arranged like brickwork, leaving no

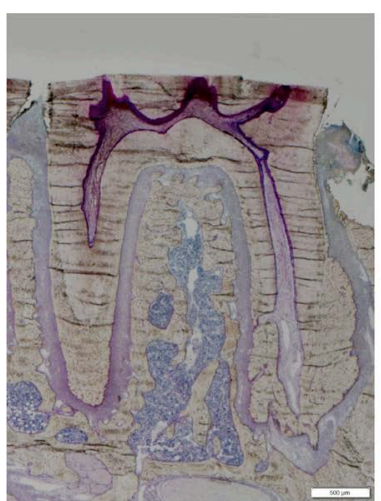

Hard diet group

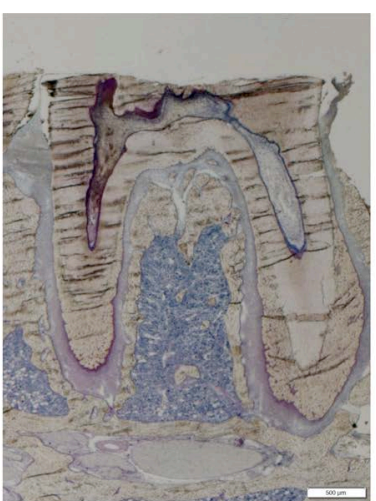

Powder diet group

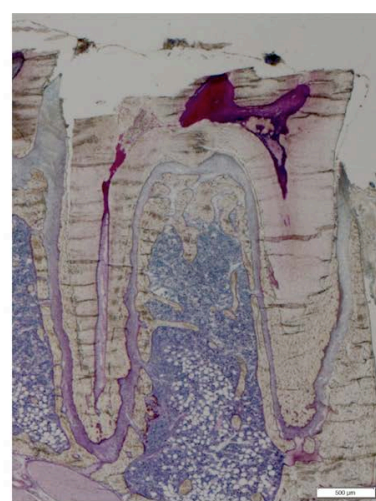

Extraction group

Figure 6. Tissue images of sagittal sections of the interradicular septum in the first mandibular molar. Bar: $500 \mu \mathrm{m}$. 
gaps. Furthermore, the height of osteoblasts [19] was increased and consistent. The Powder diet specimens also showed type II osteoblasts, but in lesser numbers. Additionally, the cell heights were shorter compared to those in the Hard diet specimens. In the extraction specimens, type IV osteoblasts were identified based on their flat cytoplasm and nuclei; the osteoblasts were lesser in number. In the control specimens, the osteoids were thick and even, while those in the experimental specimens were thinner by comparison (Figure $7(\mathrm{~b})$ ). Regarding the double bone labels, compared to controls, the intervals were shorter in the experimental groups (Figure $7(\mathrm{c})$ ). Regarding the length of the double-labeled surfaces, in the control group, many surfaces were uninterrupted, but in the experimental groups, they were shorter by comparison (Figure $7(d)$ ). ISV was greater in the control specimens than in the experimental specimens. In the control specimens, the interradicular septa had broad, sheet-like trabeculae. The trabeculae in the powder specimens were fragmentary, and those in the extraction specimens were more finely shredded (Figure 7(e)).

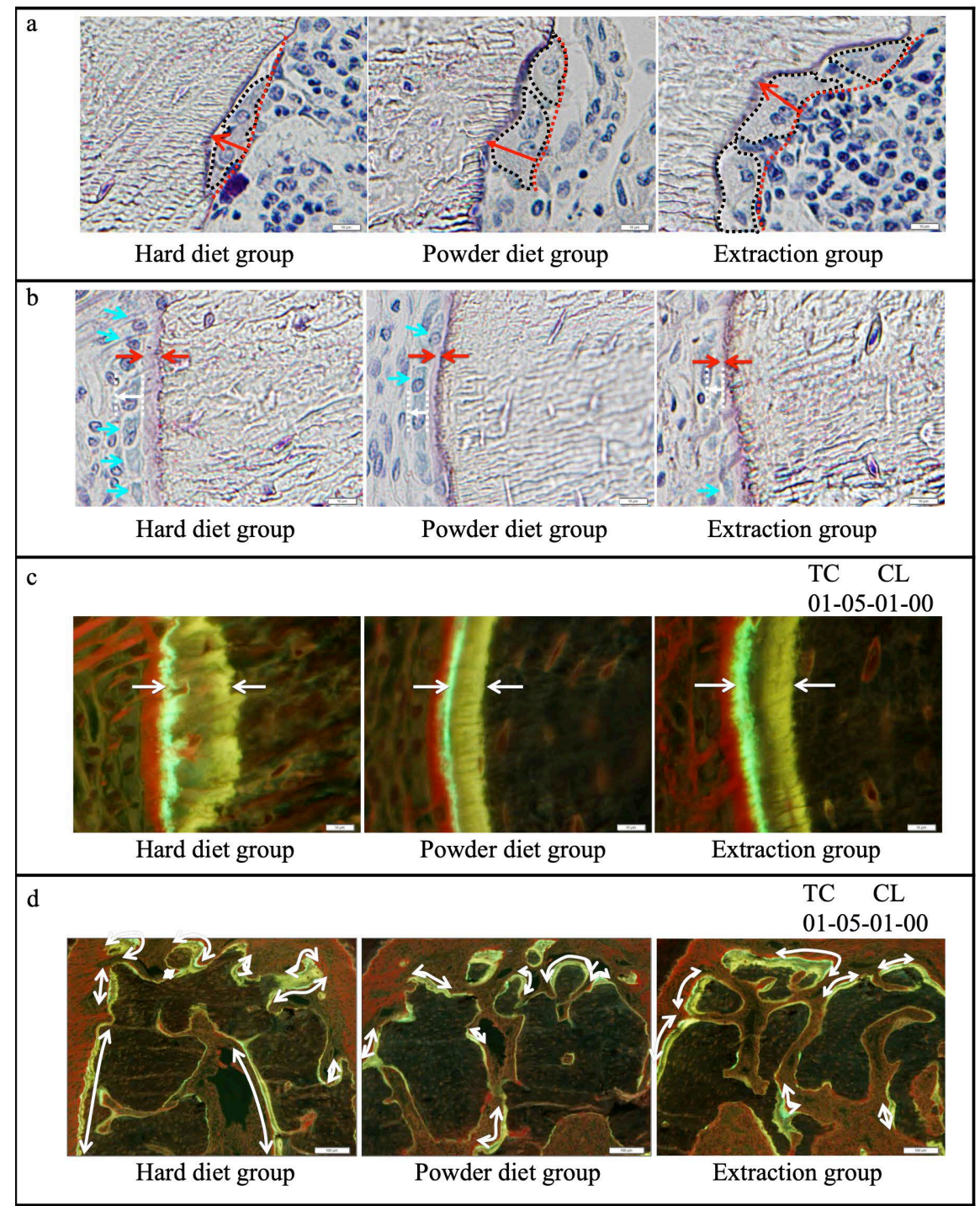




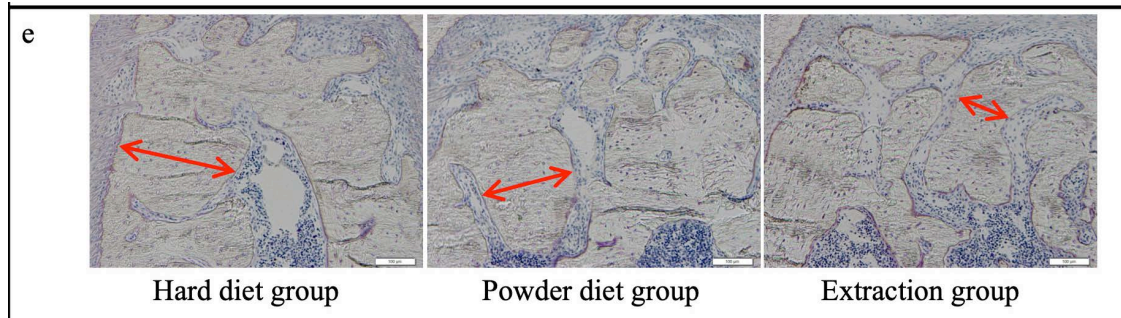

Figure 7. Tissue images of sagittal sections of the interradicular septum in the first mandibular molar; (a) The osteoclasts are shown within the dotted lines. Bone lining cells (BLCs) are seen at the apex of the osteoclasts. The red dotted line indicates the boundary between the clusters of bone marrow cells and the corresponding bone cells. The red arrows indicate the erosion depth, which is the distance to the deepest recess of the resorption cavity. Bar: $10 \mu \mathrm{m}$. Compared to controls, the experimental groups had greater erosion depths. (b) The osteoblast and osteoid thickness are shown. Blue arrows indicate osteoblasts. White arrows indicate osteoblast cell heights. Red arrows indicate osteoid thickness. Bar: $10 \mu \mathrm{m}$. Compared to controls, the experimental groups had few in number, short in height of the osteoblasts and thin osteoid. (c) Fluorescence microscopic images of the interradicular septum of the first mandibular molar. The emitted yellow and green light are tetracycline (injected seven days before imaging) and calcein (injected one day before imaging), respectively. Arrows indicates the double labeled width. Bar: $10 \mu \mathrm{m}$. Regarding the double bone labels, compared to controls, the intervals are shorter in the experimental groups. (d) Fluorescence microscopic images of the interradicular septum of the first mandibular molar. Arrows indicates the length of the double labeled surfaces. Bar: $100 \mu \mathrm{m}$. Regarding the length of the double labeled surfaces, in the control group, many surfaces continue uninterrupted, but in the experimental groups, they are shorter in comparison. (e) High magnification of the interradicular septum of the first mandibular molar. Arrows indicate the interradicular septum width. Bar: $100 \mu \mathrm{m}$. The Hard diet group had broad, sheet-like trabeculae, the Powder group had fragmented trabeculae, and the Extraction group had more finely shredded trabeculae.

\subsection{Bone Histomorphometry of Cancellous Bone in the First Molar}

We performed bone histomorphometry of the interradicular septum of the cancellous bone in the first molar (Figure 8). I.S.V, a BV parameter, was $0.78 \pm 0.04$ $\mathrm{mm}^{2}, 0.68 \pm 0.11 \mathrm{~mm}^{2}$, and $0.53 \pm 0.08 \mathrm{~mm}^{2}$ in the Hard diet group, Powder diet group, and the Extraction group, respectively. The I.S.V in the Extraction group was significantly lower than that in the Hard diet group. I.S.Wi was $140.07 \pm$ $15.43 \mu \mathrm{m}, 120.69 \pm 23.05 \mu \mathrm{m}$, and $97.19 \pm 21.51 \mu \mathrm{m}$ in the Hard diet group, Powder diet group, and the Extraction group, respectively. Moreover, I.S.Wi in the Extraction group was also significantly lower than that in the Hard diet group. O.Th, a bone formation marker, was $5.38 \% \pm 0.08 \%, 3.46 \% \pm 0.82 \%$, and $2.99 \% \pm 0.2 \%$ in the Hard diet group, Powder diet group, and Extraction group, respectively. O.Th in the Powder diet and Extraction groups were significantly lower than that in the Hard diet group. OS/BS was $34.51 \% \pm 4.52 \%, 24.42 \% \pm$ $5.01 \%$, and $23.34 \% \pm 3.44 \%$ in the Hard diet group, Powder diet group, and Extraction group, respectively. OS/BS in the Powder diet and Extraction groups were significantly lower than that in the Hard diet group. ES/BS was $9.27 \% \pm$ $3.42 \%, 19.77 \% \pm 4.34 \%$, and $29.49 \% \pm 6.52 \%$ in the Hard diet group, Powder diet 


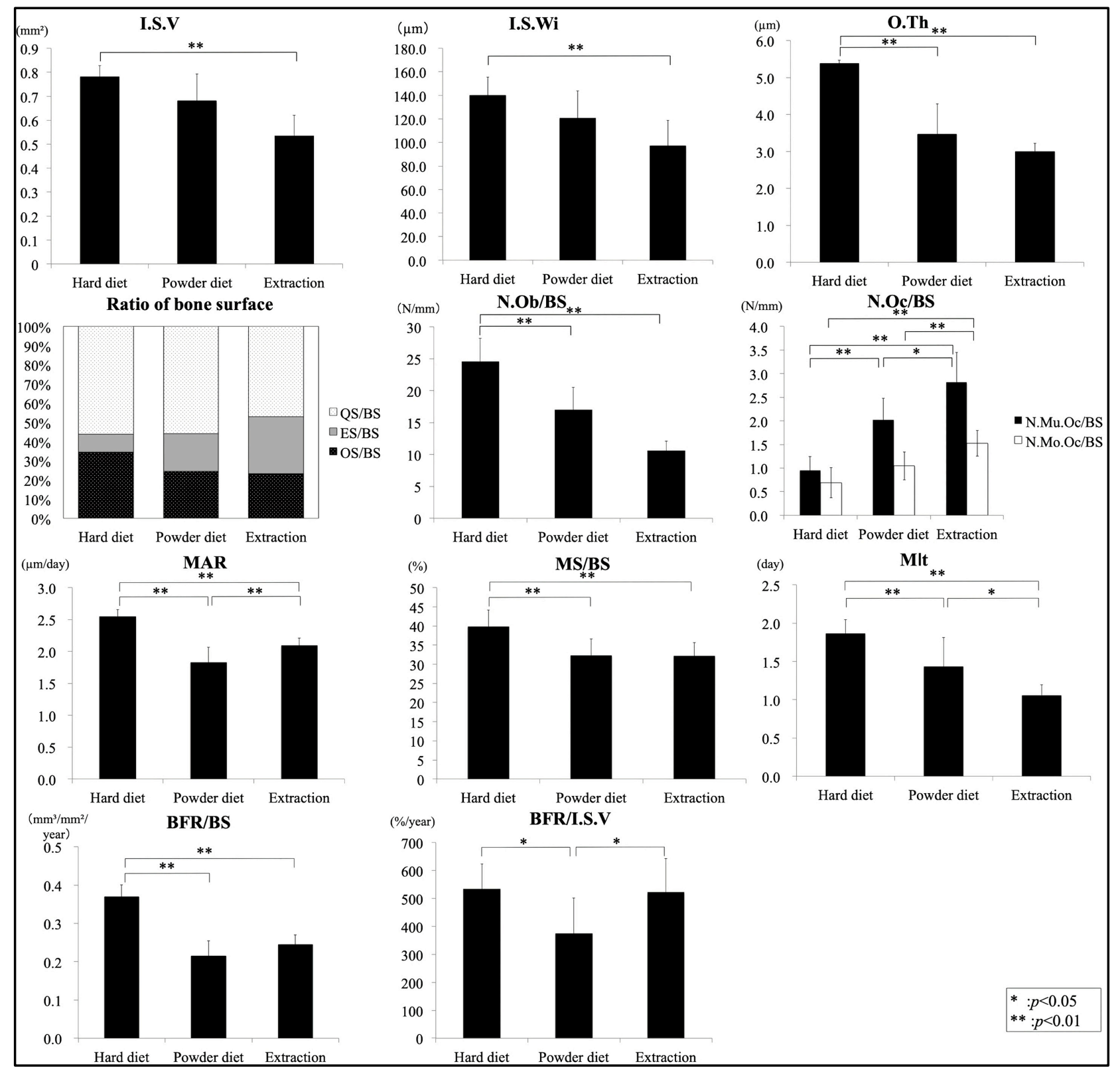

Figure 8. Histomorphometric analysis of secondary cancellous bones in the interradicular septum of the mandibular first molar. Histomorphometrical parameters (I.S.V; Interradicular septum volume, I.S.Wi; Interradicular septum width, O.Th; Osteoid thickness, ES/BS; eroded surface/bone surface, OS/BS; osteoid surface/bone surface, QS/BS; quiescent surface/bone surface, N.Ob/BS; osteoblast number/bone surface, N.Oc/BS; osteoclast number/bone surface, N.Mo.Oc/BS; mononuclear osteoclast number/bone surface, N.Mu.Oc/BS; multinuclear osteoclast number/bone surface, MAR; mineral apposition rate, MS/BS; mineralizing surface/bone surface, Mlt; mineralization lag time, BFR/BS; bone formation rate/bone surface and BFR/I.S.V; bone formation rate/Interradicular septum volume) of cancellous bone in the interradicular septum of the mandibular first molar. Data are presented as mean \pm standard deviation. $\left({ }^{\star} p<0.05\right.$ and $\left.{ }^{\star *} p<0.01\right)$

group, and Extraction group, respectively. ES/BS in the Powder diet and Extraction groups were significantly higher than that in the Hard diet group. QS/BS was $56.20 \% \pm 5.30 \%, 55.79 \% \pm 4.38 \%$, and $47.16 \% \pm 4.52 \%$ in the Hard diet group, Powder diet group, and Extraction group, respectively. QS/BS in the Extraction group was significantly lower than that in the Powder diet and Hard di- 
et groups. N.Ob/BS was $24.57 \pm 3.65 \mathrm{~N} / \mathrm{mm}$ in the Hard diet group, $16.99 \pm 3.55$ $\mathrm{N} / \mathrm{mm}$ in the Powder diet group, and $10.56 \pm 1.51 \mathrm{~N} / \mathrm{mm}$ in the Extraction group. N.Ob/BS in the Powder diet and Extraction groups were significantly lesser than that in the Hard diet group. N.Mu.Oc/BS, a bone resorption parameter, was $0.94 \pm 0.29 \mathrm{~N} / \mathrm{mm}, 2.02 \pm 0.45 \mathrm{~N} / \mathrm{mm}$, and $2.81 \pm 0.63 \mathrm{~N} / \mathrm{mm}$ in the Hard diet group, Powder diet group, and Extraction group, respectively. N.Mu.Oc/BS in the Powder diet and Extraction groups were significantly higher than that in the Hard diet group. N.Mo.Oc/BS was $0.69 \pm 0.32 \mathrm{~N} / \mathrm{mm}, 1.04 \pm$ $0.29 \mathrm{~N} / \mathrm{mm}$, and $1.52 \pm 0.27 \mathrm{~N} / \mathrm{mm}$ in the Hard diet group, Powder diet group, and Extraction group, respectively. N.Mo.Oc/BS in the Extraction group was significantly higher than that in the Powder diet and the Hard diet groups. MAR, a kinetic parameter, was $2.54 \pm 0.11 \mu \mathrm{m} /$ day, $1.83 \pm 0.23 \mu \mathrm{m} /$ day, and $2.09 \pm 0.11$ $\mu \mathrm{m} /$ day in the Hard diet group, Powder diet group, and Extraction group, respectively. MAR in the Powder diet and Extraction groups were significantly lesser than that in the Hard diet group. MS/BS was $39.88 \% \pm 4.21 \%, 32.23 \% \pm$ $4.35 \%$, and $32.1 \% \pm 3.55 \%$ in the Hard diet group, Powder diet group, and Extraction group, respectively. MS/BS in the Extraction group was significantly lower than that in the Powder diet and Hard diet groups. Mlt was $1.86 \pm 0.18$ day, $1.43 \pm 0.38$ day, and $1.05 \pm 0.13$ day in the Hard diet group, Powder diet group, and Extraction group, respectively. Mlt in the Powder diet and Extraction groups were significantly lesser than that in the Hard diet group. BFR/BS was $0.36 \pm 0.03 \mathrm{~mm}^{3} / \mathrm{mm}^{2} /$ year, $0.21 \pm 0.03 \mathrm{~mm}^{3} / \mathrm{mm}^{2} /$ year, and $0.24 \pm 0.02$ $\mathrm{mm}^{3} / \mathrm{mm}^{2} /$ year in the Hard diet group, Powder diet group, and Extraction group, respectively. BFR/BS in the Extraction group was significantly lesser than that in the Hard diet and Powder diet groups. BFR/I.S.V was 535.18 \pm 87.32\%/year, $375.29 \pm 126.18 \%$ /year, and 523.57 $\pm 119.28 \%$ /year in the Hard diet group, Powder diet group, and Extraction group, respectively. BFR/ISV in the Powder diet group was significantly lesser than that in the Extraction and Hard diet groups.

In summary, compared to controls, the experimental groups had lower values of bone mass parameters (I.S.V, I.S.Wi), bone formation parameters (O.Th, OS/BS, N.Ob/BS), and bone mineralization parameters (MAR, MS/BS, Mlt, $\mathrm{BFR} / \mathrm{BS}$ ), and higher values of bone resorption parameters (ES/BS, N.Oc/BS), and the difference from the control group were greater in the Extraction group than in the Powder diet group.

\section{Discussion}

Many studies have suggested the influence of masticatory functional loss on morphology, bone mass and bone density of alveolar bone; however, remodeling of alveolar bone has not been defined. We aimed to examine how soft diet and tooth loss in growing rats affected alveolar bone turnover. In this study, we have shown that soft diet and tooth loss may cause a worsening of bone microstructure, reduced osteogenesis, and promotion of bone resorption in alveolar bone.

We used rats as test subjects because they mature quickly, allowing us to ad- 
minister feed to many subjects under controlled conditions. Another reason was that many studies have used rats to examine the craniofacial effects of a soft diet. According to one such study, the occlusal force was approximately $400 \mathrm{~g}$ during biting [20]. Another study indicated that active tetanic tension in the masseter muscle was significantly lower in rats fed with a soft diet compared to in those fed with a hard diet [21]. Similarly, at higher activity levels, the duty time of the superficial masseter muscle in the soft diet group was significantly lower than that in the Hard diet group [22].

In light of these findings, we prepared a powdered feed, which could be ingested without chewing or crushing, and fed it to rats in the experimental groups (Powder diet group and Extraction group) in order to minimize masticatory stimulation to the alveolar bone and to reduce activity in the masticatory muscles. In the Extraction group, we reduced masticatory stimulation to the mandibular alveolar bone even further by extraction of molars (in addition to feeding a powder diet), simulating a case of congenital hypodontia or premature tooth loss caused by dental trauma or dental caries. Previous studies showed that in contrast to that in the mandible, the values of BV/TV, Trabecular thickness, trabecular number, trabecular width, and trabecular star volume in the maxillary alveolar bone were not significantly changed in rats fed a soft diet when compared with those fed a hard diet [23]. Therefore, in this study, we extracted the maxillary molars and observed the mandibular alveolar bone. In addition, the interradicular alveolar bone in the M1 was chosen because this area is exposed to concentrated occlusal stimuli and is often used for alveolar bone histomorphometry [23] [24].

Extraction was performed at 4 weeks of age, which corresponds to eruption of the third molars. We evaluated all animals at the age of 20 weeks, when the development of mandibular bone has been reported to be complete [7]. In many studies [15] [17] [25] [26] [27], rats with extracted molars were fed hard diet. However, we fed a soft diet in this study because we wanted to reduce the load on the mandibular molar.

We observed no inter-group differences in body weight of the rats at any point. Body weight increased over time in all three groups. This finding denoted that powdered diet and tooth loss had no physiological effect on rats. Physiological condition can therefore be excluded as a reason for the mandibular changes observed in the experimental groups.

On the three-dimensional constructed images, both the Extraction group and the Powder diet group showed expansion of the medullary cavity of the interradicular septum in the first molar compared to the Hard diet group. The medullary cavities were particularly large in the Extraction group, as revealed by the size of the area in the transmission image. Our data were broadly consistent with findings of studies in rats fed a soft diet or with extracted antagonist tooth [23] [25]. Literature indicates that a soft diet or extraction of the antagonist tooth can cause higher alveolar bone [10] [12] [14] [15]. In our study, we used CT analysis to determine the alveolar type based on size, as we aimed to evaluate the can- 
cellous bone without influence of the size in the interradicular septa of the first mandibular molars. The analysis revealed that BV, BMC, and BMD were significantly lower in the experimental groups than in the control group, with the difference from control group being greater in the Extraction group. Regarding the BV in rats fed with a soft diet, similar results were reported [16] [23] [28]. Similarly, in the alveolar bone that extracted antagonist tooth, the decrease in BV was reported [15] [25] [27]. Some studies have suggested that the mineral density in rats fed with a soft diet was significantly increased compared to in those fed with a hard diet [5] [29] [30]. They measured the cortical portion of the alveolar bone, at an early stage. As we measured the cancellous bone of the interradicular septum in the first molar after 17 weeks in this study, we considered that a different finding was obtained. A lower cancellous BMD in the mandibular first molar was reported in rats, which caused masticatory hypofunction, by the insertion of a bite-opening appliance and soft diet [13] [31]. Our findings are consistent with the results of these studies. We hypothesized that the experimental groups in our study had a lower BV, BMC, and BMD because masticatory stimulation to the mandibular alveolar bone was inhibited by the soft diet, and inhibited further by tooth loss in the Extraction group, leading to expansion of the bone medullary cavity, sparser trabecular density, and delay in bone deposition. We used a histological analysis to further clarify this causal relationship.

\subsection{Bone Mass}

On histo-morphometric analysis, the values of the bone mass markers (I.S.V, I.S.Wi) were lower in the experimental groups compared to controls. Regarding ISV, based on tissue imaging, the control specimens had broad, sheet-like trabeculae, powder specimens were fragmented trabeculae, and extraction specimens had more finely shredded trabeculae. Similar findings were observed for I.S.Wi.

Another finding on tissue imaging was that the interradicular septal outline was thin and broken in several places in the experimental groups, while it was thick and unbroken in the control group. In their study on rats, Bresin et al. [16] found that a soft diet was associated with reduced cortical thickness below and lateral to the first molar, which is consistent with our observation in the septal outline. The septal outline was thinner and trabeculae were fragmentary in the experimental groups because masticatory stimulation was reduced by a soft diet and, in the Extraction group, tooth loss.

\subsection{Bone Formation}

The values of the bone formation markers (OS/BS, O.Th, N.Ob/BS) were significantly lower in the experimental groups. Our results are consistent with those of previous study. Ejiri et al. [15] have reported that bone formation was significantly suppressed in the alveolar bone by antagonist tooth extraction. Gerard et al. [17] reported that 15 days after antagonist tooth extraction, the periodontal 
ligament was obviously narrowed, its structure was disorganized, and woven bone formation was noted at the top of the interradicular septa, beneath the sockets along their region of modeling. This is thought to be related to tooth extrusion due to antagonist tooth extraction. Johnson et al. [26] also reported that after 1 to 5 weeks of antagonist tooth extraction, new bone formation was observed in the alveolar bone between the first and second molar in the Extraction group than the control group. The new bone mass likely resulted from supraeruption of the second molar, which displaced the mucoperiosteum and increased the strain delivered to crestal alveolar bone by alveolar crest [32]. In this study, extrusion of the second molar was observed in some individuals; however, an increase in bone formation markers was not detected because of a prolonged duration after antagonist tooth extraction. We identified type II (cuboidal) osteoblasts, based on their cubic cytoplasm, in the Hard diet and Powder diet groups. In the Extraction group, we identified type IV osteoblasts based on their flat cytoplasm and nuclei. We also found that the height of the osteoblasts was maximum in the Hard diet group. Cell height was small in the Powder diet group, and even smaller in the Extraction group as compared with that in the Hard diet group. Type II refers to "active" osteoblasts, and type IV refers to "inactive" osteoblasts. Morphologically active osteoblasts synthesize the bone matrix more rapidly than morphologically inactive osteoblasts [18]. It is suggested that the reduced masticatory stimulation resulted in a slower formation of the bone matrix as well as a decrease in the numbers of osteoblasts and osteoids.

\subsection{Resorption}

The values of the bone resorption markers (ES/BS, N.Oc/BS) were significantly higher in the experimental groups. The Extraction group had a particularly high count of large, polynuclear osteoclasts, suggesting deep resorption cavities. Although mononuclear osteoclasts can resorb bone, large, polynuclear osteoclasts account for most osteoclasts actively engaged in bone resorption. Therefore, bone resorption would have been rampant in the Extraction group. Similarly, Honda et al. [25] reported increased N. Oc in the alveolar bone of extracted antagonist tooth. In another study, Enokida et al. [24] fed rats a soft diet and used a metal device to prevent occlusion. They reported that these conditions led to increased N. Oc in the interradicular alveolar bone in the mandibular M1. Thus, reduced masticatory stimulation facilitated resorption as well as reduced the number of osteoclasts.

\subsection{Bone Mineralization}

Among the bone mineralization markers (MAR, MS/BS, Mlt, BFR/BS, BFR/I.S.V), MAR was lower in the experimental groups. This may have occurred because of the increase in osteoclastic resorption, which uncoupled the osteoblast-osteoclast equilibrium, resulting in lesser osteoblastic mineralization. MAR was higher in the Extraction group than in the Powder diet group. Additionally, ES/BS and 
N.Oc/BS showed similar results. In the Extraction group, this may have occurred because of an increase in osteoclastic resorption, which coupled the osteoblast-osteoclast equilibrium, resulting in higher mineral apposition rate. MS/BS was significantly lower in the experimental groups. Tissue imaging revealed an inter-group difference in length of the double-labeled surfaces: in the control group, many surfaces continued uninterrupted, but in the experimental groups, they were shorter in comparison. We used two measures for bone formation rate: the bone formation rate/bone surface (BFR/BS) and the bone formation rate/interradicular septum (BFR/I.S.V). Both measures were lower in the Powder diet group than in the Hard diet group. This result is attributable to the extremely low MS/BS and MAR in the Powder diet group. On the other hand, in the Extraction group, BFR/I.S.V was similar to that in the Hard diet group. This result is attributable to the fact that the Extraction group had low MS/BS and MAR and markedly low I.S.V. The Mlt indicated a time lag between matrix production and mineralization; the values were significantly lower in the experimental groups. Therefore, histological analysis revealed that reduced masticatory stimulation was associated with a decline in the four bone mineralization markers (MAR, MS/BS, Mlt, BFR/BS).

These differences might be explained by the Wolff's Law. Julius Wolff, after discovering that trabecular architecture reflects the mechanical environment, postulated that a bone's architecture adjusts to the mechanical load under which the bone is placed, such that the bone shape and mass are commensurate with the load [33]. Thus, because the mechanical load on the alveolar bone differed between the three groups, each group developed a different trabecular architecture, adapted to withstand the particular load for that group.

Offering further insights into the results, Harold Frost, as part of his mechanostat theory, postulated that mechanical stress shapes bone structure [34] [35] [36]. Frost argued that moderate loading facilitates bone formation, while reduced loading facilitates bone resorption. Mechanical stress on the alveolar bone was low in the two experimental groups due to the powdered feed, and particularly low in the Extraction group due to the tooth loss. Hence, bone resorption was greater in the experimental groups than in the control group. This understanding is corroborated by the CT results, which indicate lower BV, BMC, and BMD values in the experimental groups.

For bone remodeling, osteoclastic resorption precedes osteoblastic formation, osteoclasts are attached to the bone matrix and initiate resorption, and the osteoblasts initiate bone formation and mineralization. Repeated iterations of resorption followed by formation determine the bone mass in the interradicular septum. Taken together, the results of our study imply that a loss in masticatory stimulation increases the number of osteoclasts in the alveolar bone, such that each iteration of the bone remodeling cycle, the osteoblasts (proceeding the osteoclasts in said cycle) operate to a lesser extent than the osteoclasts. This significant resorption uncoupling in the osteoblast-osteoclast equilibrium results in 
reduced interradicular septal bone mass. Thus, masticatory mechanical stress to the alveolar bone plays a significant role in sustaining interradicular septal bone mass. Individuals who prefer a soft diet (as is increasingly the case today) or with congenital hypodontia or premature tooth loss, are more likely to have reduced bone mass in their interradicular septum, as a result of the significant resorption uncoupling in the osteoblast-osteoclast equilibrium. Therefore, prevention of dental trauma and dental caries, and early detection and treatment of dental caries are important to prevent premature tooth loss. In the case of congenital hypodontia or premature tooth loss, pediatric denture should be attached to recover the occlusal function. In addition, a well-balanced diet without frequent eating of soft diet should be recommended. Appropriate masticatory stimulation plays an important role in the regulation of the osteoblast-osteoclast equilibrium and the maintenance of functional alveolar structure. This study examined the long-term bone turnover. In the future, we plan to conduct further studies to clarify the influence of early stage of reduced masticatory stimulation on alveolar bone turnover.

\section{Conclusions}

To summarize, in our study, the experimental groups exhibited lower bone mass in the interradicular septum below the first molar, with more osteoclasts and greater osteoclastic resorption. Compared to controls, the experimental groups also showed lower values of the osteoid surface, osteoid thickness, number of osteoblasts, mineralization surface, and MAR.

Diminished masticatory stimulation (as a result of soft diet and, in the case of the experimental group, tooth loss) led to reduced bone mass in the interradicular septum because it significantly uncoupled osteoblast-osteoclast equilibrium in the alveolar bone. The number of osteoclasts increased such that the osteoblasts proceeding the osteoclasts in the bone reproduction cycle operated at a lesser extent than the osteoclasts. Therefore, we conclude that appropriate masticatory mechanical stress to the alveolar bone plays a significant role in the regulation of the osteoblast-osteoclast equilibrium and the maintenance of functional alveolar structure. Individuals who either prefer a soft diet, have congenital hypodontia, or premature tooth loss are more likely to have reduced bone mass in the interradicular septum because of the significant resorption uncoupling in the osteoblast-osteoclast equilibrium.

\section{Acknowledgements}

We would like to thank Dr. Akemi Ito (Ito Bone Histomorphometry Institute Co., Niigata, Japan) for her assistance with the histological analysis of cancellous bone and Editage (www.editage.com) for English language editing.

\section{Conflicts of Interest}

There are no conflicts of interest to declare. 


\section{References}

[1] Moss, M.L. and Meehan, M. (1970) Functional Cranial Analysis of the Coronoid Process in the Rat. Acta Anatomica, 77, 11-24. https://doi.org/10.1159/000143524

[2] Katsaros, C., Berg, R. and Kiliaridis, S. (2002) Influence of Masticatory Muscle Function on Transverse Skull Dimensions in the Growing Rat. Journal of Orofacial Orthopedics, 63, 5-13. https://doi.org/10.1007/s00056-002-9903-0

[3] Enomoto, A., Watahiki, J., Yamaguchi, T., Irie, T., Tachikawa, T. and Maki, K. (2010) Effects of Mastication on Mandibular Growth Evaluated by Microcomputed Tomography. European Journal of Orthodontics, 32, 66-70. https://doi.org/10.1093/ejo/cjp060

[4] Hichijo, N., Kawai, N., Mori, H., Sano, R., Ohnuki, Y., Okumura, S., Langenbach, G.E.J. and Tanaka, E. (2014) Effects of the Masticatory Demand on the Rat Mandibular Development. Journal of Oral Rehabilitation, 41, 581-587. https://doi.org/10.1111/joor.12171

[5] Hichijo, N., Tanaka, E., Kawai, N., van Ruijven, L.J. and Langenbach, G.E.J. (2015) Effects of Decreased Occlusal Loading during Growth on the Mandibular Bone Characteristics. PLoS ONE, 10, e0129290. https://doi.org/10.1371/journal.pone.0129290

[6] Abed, G.S., Buschang, P.H., Taylor, R. and Hinton, R.J. (2007) Maturational and Functional Related Differences in Rat Craniofacial Growth. Archives of Oral Biology, 52, 1018-1025. https://doi.org/10.1016/j.archoralbio.2007.05.008

[7] Muramatsu, H., Zhang, X. and Ogawa, K. (2012) Jawbone Morphology in Rats with Extracted Maxillary Molars Reared on Powdered Diet. International Journal of Oral-Medical Sciences, 11, 211-217.

[8] Abo, N., Ogawa, K. and Shimizu, K. (2013) Craniofacial Development in Rats Fed on Powdered Diet following Extraction of All Upper Molars. International Journal of Oral-Medical Sciences, 12, 129-140.

[9] Ogawa, K., Kiguchi, Y., Yamamoto-Nemoto, S., Hirai, N., Sawamoto, K. and Shimizu, T. (2016) Loss of Masticatory Function Affects Growth and Development of the Mandibular Condyle in Rats. Open Journal of Stomatology, 6, 261-273. https://doi.org/10.4236/ojst.2016.612032

[10] Mavropoulos, A., Odman, A., Ammann, P. and Kiliaridis, S. (2010) Rehabilitation of Masticatory Function Improves the Alveolar Bone Architecture of the Mandible in Adult Rats. Bone, 47, 687-692. https://doi.org/10.1016/j.bone.2010.06.025

[11] Bresin, A., Johansson, C.B. and Kiliaridis, S. (1994) Effects of Occlusal Strain on the Development of the Dentoalveolar Process in the Growing Rat: A Morphometric Study. European Journal of Experimental Musculoskeletal Research, 3, 112-122.

[12] Watt, D.G. and Williams, C.H.M. (1951) The Effects of the Physical Consistency of Food on the Growth and Development of the Mandible and the Maxilla of the Rat. American Journal of Orthopedics, 37, 895-928. https://doi.org/10.1016/0002-9416(51)90101-7

[13] Mavropoulos, A., Kiliaridis, S., Bresin, A. and Ammann, P. (2004) Effect of Different Masticatory Functional and Mechanical Demands on the Structural Adaptation of the Mandibular Alveolar Bone in Young Growing Rats. Bone, 35, 191-197. https://doi.org/10.1016/j.bone.2004.03.020

[14] Odman, A., Mavropoulos, A. and Kiliaridis, S. (2008) Do Masticatory Functional Changes Influence the Mandibular Morphology in Adult Rats. Archives of Oral Biology, 53, 1149-1154. https://doi.org/10.1016/j.archoralbio.2008.07.004 
[15] Ejiri, S., Toyooka, E., Tanaka, M., Anwar, R.B. and Kohno, S. (2006) Histological and Histomorphometrical Changes in Rat Alveolar Bone Following Antagonistic Tooth Extraction and/or Ovariectomy. Archives of Oral Biology, 51, 941-950. https://doi.org/10.1016/j.archoralbio.2006.05.006

[16] Bresin, A., Kiliaridis, S. and Strid, K.G. (1999) Effect of Masticatory Function on the Internal Bone Structure in the Mandible of the Growing Rat. European Journal of Oral Sciences, 107, 35-44. https://doi.org/10.1046/j.0909-8836.1999.eos107107.x

[17] Levy, G.G. and Mailland, M.L. (1980) Histologic Study of the Effects of Occlusal Hypofunction Following Antagonist Tooth Extraction in the Rat. Journal of Periodontology, 51, 393-399. https://doi.org/10.1902/jop.1980.51.7.393

[18] Villanueva, A.R., Mathews, C.H.E. and Perfitt, A.M. (1997) Relationship between the Size and Shape of Osteoblasts and the Width of Osteoid Seams in Bone. In: Takahashi, E., Ed., Handbook of Bone Morphometry, 2nd Edition, Nishimura Co., Ltd., Niigata, 69-73.

[19] Katayama, Y., Battista, M., Kao, W., Hidalgo, A., Peired, A.J., Thomas, S.A. and Frenette, P.S. (2006) Signals from the Sympathetic Nervous System Regulate Hematopoietic Stem Cell Egress from Bone Marrow. Cell, 124, 407-421. https://doi.org/10.1016/j.cell.2005.10.041

[20] Robins, M.W. (1977) Biting Loads Generated by the Laboratory Rat. Archives of Oral Biology, 22, 43-47. https://doi.org/10.1016/0003-9969(77)90138-8

[21] Kiliaridis, S. and Shyu, B.C. (1988) Isometric Muscle Tension Generated by Masseter Stimulation after Prolonged Alteration of the Consistency of the Diet Fed to Growing Rats. Archives of Oral Biology, 33, 467-472, https://doi.org/10.1016/0003-9969(88)90026-X

[22] Kawai, N., Sano, R., Korfage, J.A.M., Nakamura, S., Kinouchi, N., Kawakami, E., Tanne, K., Langenbach, G.E.J. and Tanaka, E. (2010) Adaptation of Rat Jaw Muscle Fibers in Postnatal Development with a Different Food Consistency: An Immunohistochemical and Electromyographic Study. Journal of Anatomy, 216, 717-723. https://doi.org/10.1111/j.1469-7580.2010.01235.x

[23] Shimizu, Y., Ishida, T., Hosomichi, J., Kaneko, S., Hatano, K. and Ono, T. (2013) Soft Diet Causes Greater Alveolar Osteopenia in the Mandible than in the Maxilla. Archives of Oral Biology, 58, 907-911. https://doi.org/10.1016/j.archoralbio.2013.02.003

[24] Enokida, M., Kaneko, S., Yanagishita, M. and Soma, K. (2005) Influence of Occlusal Stimuli on the Remodelling of Alveolar Bone in a Rat Hypofunction-Recovery Model. Journal of Oral Biosciences, 47, 321-334. https://doi.org/10.1016/S1349-0079(05)80015-5

[25] Honda, K., Watari, I., Takei, M. and Ono, T. (2011) Changes in the Microstructure of the Rat Alveolar Bone Induced by Unilateral Molar Extraction and Estrogen Deficiency. Orthodontic Waves, 70, 143-150. https://doi.org/10.1016/j.odw.2011.07.001

[26] Johnson, R.B. (1990) Effect of Altered Occlusal Function on Transseptal Ligament and New Bone Thicknesses in the Periodontium of the Rat. American Journal of Anatomy, 187, 91-97. https://doi.org/10.1002/aja.1001870110

[27] von Wowern, N., Hjørting-Hansen, E. and Stoltze, K. (1979) Changes in Bone Mass in Rat Mandibles after Tooth Extraction. International Journal of Oral Surgery, 8, 229-233. https://doi.org/10.1016/S0300-9785(79)80024-1

[28] Kiliaridis, S., Bresin, A., Holm, J. and Strid, K.G. (1996) Effects of Masticatory Muscle Function on Bone Mass in the Mandible of the Growing Rat. Acta Anatomica, 
155, 200-205. https://doi.org/10.1159/000147805

[29] Kingsmill, V.J., Boyde, A., Davis, G.R., Howell, P.G. and Rawlinson, S.C.F. (2010) Change in Bone Mineral and Matrix in Response to a Soft Diet. Journal of Dental Research, 89, 510-514. https://doi.org/10.1177/0022034510362970

[30] Tanaka, E., Sano, R., Kawai, N., Langenbach, G.E., Brugman, P., Tanne, K. and van Eijden, T.M. (2007) Effect of Food Consistency on the Degree of Mineralization in the Rat Mandible. Annals of Biomedical Engineering, 35, 1617-1621.

https://doi.org/10.1007/s10439-007-9330-x

[31] Sato, H., Kawamura, A., Yamaguchi, M. and Kasai, K. (2005) Relationship between Masticatory Function and Internal Structure of the Mandible Based on Computed Tomography Findings. American Journal of Orthodontics and Dentofacial Orthopedics, 128, 766-773. https://doi.org/10.1016/j.ajodo.2005.05.046

[32] Rippin, J.W. (1976) Collagen Turnover in Periodontal Ligament under Normal and Altered Functional Forces: Young Rat Molars. Journal of Periodontal Research, 11, 101-107. https://doi.org/10.1111/j.1600-0765.1976.tb00057.x

[33] Wolff, J. (1986) The Law of Bone Remodeling. Springer, Berlin. https://doi.org/10.1007/978-3-642-71031-5

[34] Frost, H.M. (1987) Bone "Mass" and the "Mechanostat": A Proposal. The Anatomical Record, 219, 1-9. https://doi.org/10.1002/ar.1092190104

[35] Frost, H.M. (2003) Bone's Mechanostat: A 2003 Update. The Anatomical Record, 275, 1081-1101. https://doi.org/10.1002/ar.a.10119

[36] Frost, H.M. (2004) A 2003 Update of Bone Physiology and Wolff's Law for Clinicians. The Angle Orthodontics, 74, 3-15. 